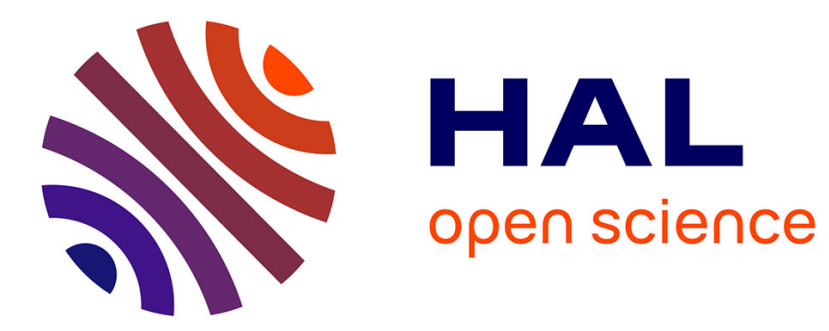

\title{
Mathematical Analysis of a Inf-Convolution Model for Image Processing
}

\author{
Maïtine Bergounioux
}

\section{To cite this version:}

Maïtine Bergounioux. Mathematical Analysis of a Inf-Convolution Model for Image Processing. Journal of Optimization Theory and Applications, 2015, 20 p. hal-01002958v3

\section{HAL Id: hal-01002958 https://hal.science/hal-01002958v3}

Submitted on 3 Apr 2015

HAL is a multi-disciplinary open access archive for the deposit and dissemination of scientific research documents, whether they are published or not. The documents may come from teaching and research institutions in France or abroad, or from public or private research centers.
L'archive ouverte pluridisciplinaire HAL, est destinée au dépôt et à la diffusion de documents scientifiques de niveau recherche, publiés ou non, émanant des établissements d'enseignement et de recherche français ou étrangers, des laboratoires publics ou privés. 
Noname manuscript No.

(will be inserted by the editor)

\title{
Mathematical Analysis of a Inf-Convolution Model for Image Processing
}

\section{Bergounioux}

the date of receipt and acceptance should be inserted later

\begin{abstract}
We deal with a second order image decomposition model to perform denoising and texture extraction that was previously presented. We look for the decomposition of an image as the summation of three different order terms. For highly textured images the model gives a two-scale texture decomposition: the first order term can be viewed as a macro-texture (larger scale) which oscillations are not too large and the zero-order term is the micro-texture (very oscillating) that contains the noise. Here, we perform mathematical analysis of the model and give qualitative properties of solutions using the dual problem and inf-convolution formulation.
\end{abstract}

Keywords Second order total variation - image decomposition · variational method · inf-convolution

Mathematics Subject Classification (2000) 65D18 $68 \mathrm{U} 10 \cdot 65 \mathrm{~K} 10$

\section{Introduction}

The most famous variational denoising model is the Rudin-Osher-Fatemi [1] one. This model involves a regularization term that preserves discontinuities, what a classical Sobolev type -Tychonov regularization method does not. The observed image to recover is split in two parts : one represents the oscillating component (noise or texture) and the other one is a smooth part, namely a function of bounded variation [2-4]. The regularization term involves only this so-called cartoon component, while the remainder term represents the noise to be minimized.

A lot of people have investigated such decomposition models based on variational formulation, considering that an image can be decomposed into

Université d'Orléans

UFR Sciences, Math., Labo. MAPMO, UMR 7349, Route de Chartres, BP 6759, 45067

Orléans cedex 2,

E-mail: maitine.bergounioux@univ-orleans.fr 
many components, each component describing a particular property of the image ([5-10] and references therein for example).

In $[11,12]$ we have presented second order models where the (first order) classical total variation term has been replaced by a second order total variation term with the appropriate functional framework, namely the space of functions with bounded hessian introduced in [13] (see also $[11,12,14]$ ). The use of such a model allows to get rid of the staircasing effect that appears with the ROF model in denoising processes. To achieve this goal K. Bredies and al. have recently introduced a second order generalized total variation definition [15-17] which is a nice compromise/mixture between the first and second order derivatives. It is, in some sense, an extension of the inf-convolution (we recall the definition later) of the first and second order derivatives. The model we present here is not more efficient than the Total Generalized Variation aproach for denoising. However, it provides a decomposition of the image at different scales what the other model does not a priori. This paper is focused on this decomposition that provides a multiscale description of textured images. Second order models have also been investigated in the context of segmentation and inpainting problems with Mumford-Shah types functionals (see [18-20] for example). The functional framework is the so called General Special Bounded Variation function space composed of functions whose truncated forms belong to the classical space of locally special bounded variation functions.

The aim of this paper is to give an existence result without the penalization term that was added in [11] and to perform a qualitative analysis of the model. Uniqueness and regularity issues will also be addressed.

More precisely, we assume that an image can be split in three components: a smooth (continuous) part $v$, a cartoon (piecewise constant) part $u$ and an oscillating part $w$ that should involve noise and/or fine textures. Such decompositions have already been investigated by Aujol and al. [5,6]. These authors use the Meyer space of oscillating functions [21] rather than the space of functions of bounded hessian (we shall present these spaces in the sequel). The model we deal with, is different: the oscillating part of the image is not penalized included but a priori in the remainder term $w=u_{d}-u-v$, while $v$ is the smooth (bounded hessian) part and $u$ belongs to the space of functions of bounded variation: we hope $u$ to be piecewise constant so that its jump set gives the image contours. This model has been proposed (in an abstract form) in [22]. However, no focus was made on the first and second order total variation. For highly textured images, the model provides a two-scale texture decomposition: $u$ can be viewed as a macro-texture (large scale) whose oscillations are not too large and $w$ is the micro-texture (much more oscillating) that contains the noise.

The paper is organized as follows. We first present the functional framework and perform a quick comparison between the second-order total variation we use and the one defined by Bredies et al. in [15]. In section 3, we present the variational model, give existence result and an equivalent formulation with infconvolution. This allows to compute the dual problem. Next section is devoted to giving qualitative properties of the solutions. 


\section{Functional Framework for Second Order Variational Analysis}

\subsection{Spaces $\mathrm{BV}(\Omega)$ and $\mathrm{BH}(\Omega)$}

In the whole paper, $\Omega$ is an open bounded subset of $\mathbb{R}^{d}$ (practically $d=2$ ) smooth enough (with the cone property and Lipschitz for example). More precisely, if $d=2, \Omega$ may satisfy next assumption

$\left\{\begin{array}{c}\Omega \text { is a bounded connected open set, strongly Lipschitz such that } \\ \partial \Omega \text { is the union of finitely many } \mathcal{C}^{2} \text { curves . }\end{array}\right.$

Following $[2,3,23]$ and $[12,13]$, we recall the definitions and main properties of the spaces of functions of first and second order bounded variation. The space $\operatorname{BV}(\Omega)$ is the classical Banach space of functions of bounded variation defined by

$$
\mathrm{BV}(\Omega):=\left\{u \in \mathrm{L}^{1}(\Omega): \operatorname{TV}(u)<+\infty\right\},
$$

where $\operatorname{TV}(u)$ is the total variation of $u$

$$
\operatorname{TV}(u):=\sup \left\{\int_{\Omega} u(x) \operatorname{div} \xi(x) d x: \xi \in \mathcal{C}_{c}^{1}\left(\Omega, \mathbb{R}^{d}\right),\|\xi\|_{\infty} \leq 1\right\},
$$

endowed with the norm $\|u\|_{\mathrm{BV}(\Omega)}:=\|u\|_{\mathrm{L}^{1}}+\mathrm{TV}(u)$.

We say that a sequence $\left(u_{n}\right)_{n \in \mathbb{N}}$ of $\mathrm{BV}(\Omega)$ converges to some $u \in \mathrm{BV}(\Omega)$ for the intermediate (or strict) convergence, if $u_{n}$ strongly converges to $\mathrm{u}$ for the $\mathrm{L}^{1}(\Omega)$ topology and $\mathrm{TV}\left(u_{n}\right)$ converges to $\mathrm{TV}(u)$ (in $\mathbb{R}$ ) (see $[2,3,24]$ ).

The space of functions with bounded hessian has been introduced by Demengel [13] (where it was denoted $\mathrm{BH}(\Omega)$ ). It is the space of $\mathrm{W}^{1,1}(\Omega)$ functions such that $\operatorname{TV}^{2}(u)<+\infty$, where $\mathrm{W}^{1,1}(\Omega)=\left\{u \in \mathrm{L}^{1}(\Omega): \nabla u \in \mathrm{L}^{1}(\Omega)\right\}$. Here, $\nabla u$ stands for the first order derivative of $u$ in the sense of distributions and

$$
\operatorname{TV}^{2}(u):=\sup \left\{\int_{\Omega}\langle\nabla u, \operatorname{div}(\xi)\rangle_{\mathbb{R}^{d}}: \xi \in \mathcal{C}_{c}^{2}\left(\Omega, \mathbb{R}^{d \times d}\right),\|\xi\|_{\infty} \leq 1\right\}<\infty,
$$

is the second order total variation of $u$.

In the sequel, $\operatorname{div}(\xi)=\left(\operatorname{div}\left(\xi_{1}\right), \operatorname{div}\left(\xi_{2}\right), \ldots, \operatorname{div}\left(\xi_{d}\right)\right)$ and

$$
\forall i, \xi_{i}=\left(\xi_{i, 1}, \xi_{i, 2}, \ldots, \xi_{i, d}\right) \in \mathbb{R}^{d}, \quad \operatorname{div}\left(\xi_{i}\right)=\sum_{j=1}^{d} \frac{\partial \xi_{i, j}}{\partial x_{j}} .
$$

The space $\mathrm{BH}(\Omega)$ endowed with the following norm

$$
\|f\|_{B H}:=\|f\|_{W^{1,1}}+\mathrm{TV}^{2}(f)=\|f\|_{\mathrm{L}^{1}}+\|\nabla f\|_{\mathrm{L}^{1}}+\mathrm{TV}^{2}(f),
$$

where $\mathrm{TV}^{2}$ is given by (3), is a Banach space. Note that a function $u$ belongs to $\operatorname{BH}(\Omega)$ if and only if $u \in \mathrm{W}^{1,1}(\Omega)$ and $\frac{\partial u}{\partial x_{i}} \in \operatorname{BV}(\Omega)$ for $i \in\{1, \ldots, d\}$. In particular: $\mathrm{TV}^{2}(u) \leq \sum_{i=1}^{d} \mathrm{TV}\left(\frac{\partial u}{\partial x_{i}}\right) \leq d \mathrm{TV}^{2}(u)$

We give thereafter important properties of these spaces which proofs can be found in $[2,3,12,13,25]$ for example. 


\section{Theorem 2.1 (Semi-continuity of total variation )}

$i$. The mapping $u \mapsto \mathrm{TV}(u)$ is lower semi-continuous (denoted in short lsc) from $\mathrm{BV}(\Omega)$ endowed with the $\mathrm{L}^{1}(\Omega)$ topology to $\mathbb{R}^{+}$.

ii. The operator $\mathrm{TV}^{2}$ is lower semi-continuous from $\mathrm{BH}(\Omega)$ endowed with the strong topology of $\mathrm{W}^{1,1}(\Omega)$ to $\mathbb{R}$.

Theorem 2.2 (Embedding results) Assume $d \geq 2$. Then

i. $\mathrm{BH}(\Omega) \hookrightarrow \mathrm{W}^{1, \mathrm{q}}(\Omega)$ with $q \leq \frac{d}{d-1}$, with continuous embedding. Moreover the embedding is compact if $q<\frac{d}{d-1}$. In particular

$$
\mathrm{BH}(\Omega) \hookrightarrow \mathrm{L}^{\mathrm{q}}(\Omega), \quad \forall q \in[1, \infty[, \quad \text { if } d=2 .
$$

ii. If $d=2$

$-\mathrm{BV}(\Omega) \subset \mathrm{L}^{2}(\Omega)$ with continuous embedding, and

- $\mathrm{BV}(\Omega) \subset \mathrm{L}^{\mathrm{p}}(\Omega)$ with compact embedding, for every $p \in[1,2[$.

iii. If $d=2$ and if $\Omega$ satisfies assumption (1) then $\operatorname{BH}(\Omega)(\Omega) \subset \mathcal{C}^{0}(\bar{\Omega})$.

So $\mathrm{BH}(\Omega) \subset \mathrm{H}^{1}(\Omega)$ with continuous embedding and $\mathrm{BH}(\Omega) \subset \mathrm{W}^{1,1}(\Omega)$ with compact embedding. Let us define the space $\mathrm{BV}_{0}(\Omega)$ as the space of functions of bounded variation that vanish on the boundary $\partial \Omega$ of $\Omega$. More precisely, as $\Omega$ is bounded and $\partial \Omega$ is Lipschitz, functions of $\operatorname{BV}(\Omega)$ have a trace of class $\mathrm{L}^{1}$ on $\partial \Omega[2,3,24]$, and the trace mapping $T: \mathrm{BV}(\Omega) \rightarrow \mathrm{L}^{1}(\partial \Omega)$ is linear, continuous from $\mathrm{BV}(\Omega)$ equipped with the intermediate convergence to $\mathrm{L}^{1}(\partial \Omega)$ endowed with the strong topology ([3] Theorem 10.2.2 p 386). The space $\mathrm{BV}_{0}(\Omega)$ is then defined as the kernel of $T$. It is a Banach space, endowed with the induced norm:

$$
\mathrm{BV}_{0}(\Omega):=\left\{u \in \operatorname{BV}(\Omega): \mathrm{u}_{\mid \partial \Omega}=0\right\} .
$$

In addition, if $u \in \mathrm{BH}(\Omega)$ then $\nabla u \in \mathrm{BV}(\Omega)^{\mathrm{n}}$ ( as a consequence of the definitition of $\mathrm{BH}(\Omega)$ ) and we may define the normal derivative trace operator $\nu: \mathrm{BH}(\Omega) \rightarrow \mathrm{L}^{1}(\partial \Omega)\left(\right.$ with $\left.\nu(u):=\nabla u \cdot \mathbf{n}=\frac{\partial u}{\partial n}\right)$. This operator is linear, continuous from $\mathrm{BH}(\Omega)$ (equipped with the intermediate convergence, that is the $\mathrm{W}^{1,1}$ strong convergence and the convergence of $\left.\mathrm{TV}^{2}\right)$ to $\mathrm{L}^{1}(\partial \Omega)$ endowed with the strong topology. So, we may define similarly

$$
\mathrm{BH}_{0}(\Omega):=\left\{u \in \operatorname{BH}(\Omega): \frac{\partial u}{\partial n}=0 \text { on } \partial \Omega\right\} .
$$

We set also

$$
\operatorname{BV}_{\mathrm{m}}(\Omega):=\left\{u \in \mathrm{BV}(\Omega): \int_{\Omega} u(x) d x=0\right\}
$$

and

$$
\mathrm{BH}_{\mathrm{m}}(\Omega):=\left\{u \in \mathrm{BH}(\Omega): \int_{\Omega} \frac{\partial u}{\partial x_{i}} d x=0 i=1, \cdots, d\right\}
$$


The Green's formula gives

$$
\int_{\Omega} \frac{\partial u}{\partial x_{i}} d x=-\int_{\partial \Omega} u_{i} n_{i}
$$

where $u_{i}$ is the $\mathrm{i}^{\text {th }}$ partial function with respect to the $\mathrm{i}^{\text {th }}$ coordinate and $n=\left(n_{1}, \cdots, n_{d}\right)$ is the outer normal vector. In particular, if $u=0$ on $\partial \Omega$, then $u \in \mathrm{BH}_{\mathrm{m}}(\Omega)$. At last we shall use the following result of [14]:

Lemma 2.1 Let $\Omega \subset \mathbb{R}^{n}$ be an open Lipschitz bounded set.There exist generic constants only depending on $\Omega, C_{1}, C_{2}>0$ such that

$$
\begin{aligned}
\forall u \in \mathrm{BV}_{\mathrm{m}}(\Omega) & \|u\|_{\mathrm{L}^{1}(\Omega)} \leq C_{1} \mathrm{TV}(u), \\
\forall u \in \mathrm{BH}_{0}(\Omega) \cup \mathrm{BH}_{\mathrm{m}}(\Omega) & \operatorname{TV}(u) \leq C_{2} \operatorname{TV}^{2}(u)
\end{aligned}
$$

\subsection{Comparison with the Space BGV ${ }^{2}$}

As already mentionned, a different approach of second-order total variation spaces has been set in [15]. The main difference lies in the choice of test functions for the weak variational formulation. The authors define the Total Generalized Variation of $u$ as the supremum of the duality product between $u$ and symmetric tests functions that are bounded together with their derivative. First, we note that we may define $\operatorname{TV}^{2}(u)$ in a equivalent way as following: for any $\xi=\left(\xi_{1}, \xi_{2}, \ldots, \xi_{d}\right) \in \mathcal{C}_{c}^{2}\left(\Omega, \mathbb{R}^{d \times d}\right)$ then $\operatorname{div}\left(\xi_{i}\right)=\sum_{j=1}^{d} \frac{\partial \xi_{i, j}}{\partial x_{j}}$ where $\xi_{i}=\left(\xi_{i, 1}, \xi_{i, 2}, \ldots, \xi_{i, d}\right) \in \mathbb{R}^{d}$, for every $i$. Then, we define as in [15]:

$$
\begin{aligned}
& \operatorname{div}^{2} \xi:= \sum_{i, j=1}^{d} \frac{\partial^{2} \xi_{i, j}}{\partial x_{i} \partial x_{j}} . \text { Denote } \mathcal{B}:=\left\{\xi \in \mathcal{C}_{c}^{2}\left(\Omega, \mathbb{R}^{d \times d}\right),\|\xi\|_{\infty} \leq 1\right\}, \text { then } \\
& \forall u \in \mathrm{W}^{1,1}(\Omega) \quad \operatorname{TV}^{2}(u)=\sup \left\{\int_{\Omega} u \operatorname{div}^{2} \xi d x: \xi \in \mathcal{B}\right\} .
\end{aligned}
$$

Indeed, an integration by parts gives: $\int_{\Omega} u \operatorname{div}^{2} \xi d x=-\int_{\Omega}(\nabla u, \operatorname{div} \xi)_{\mathbb{R}^{d}} d x$. Let be $\alpha=\left(\alpha_{0}, \alpha_{1}\right)>0$, we call

$$
\operatorname{TGV}_{\alpha}^{2}(u)=\sup \left\{\int_{\Omega} u \operatorname{div}^{2} \xi d x: \xi \in \mathcal{B}_{\alpha}\right\},
$$

where $\mathcal{B}_{\alpha}:=\left\{\xi \in \mathcal{K}: \xi_{i j}=\xi_{j i} \forall i, j,\|\xi\|_{\infty} \leq \alpha_{0},\|\operatorname{div} \xi\|_{\infty} \leq \alpha_{1}\right\}$. We may define $[15]$

$$
\operatorname{BGV}_{\alpha}^{2}(\Omega)=\left\{u \in \mathrm{L}^{1}(\Omega): \operatorname{TGV}_{\alpha}^{2}(u)<+\infty\right\} .
$$

Proposition 2.1 Let be $\alpha=\left(\alpha_{0}, \alpha_{1}\right)>0$. For every function $u$ in $\mathrm{W}^{1,1}(\Omega)$ we get: $\mathrm{TGV}_{\alpha}^{2}(u) \leq \alpha_{0} \mathrm{TV}^{2}(u)$. Therefore

$$
\forall \alpha>0 \quad \operatorname{BH}(\Omega) \subset \operatorname{BGV}_{\alpha}^{2}(\Omega)
$$

with continuous embedding. 
Proof. As $\mathcal{B}_{\alpha} \subset \alpha_{0} \mathcal{B}$ the first relation is obvious. Moreover if $u \in \mathrm{BH}(\Omega)$, then $u \in \mathrm{W}^{1,1}(\Omega)$ and $\operatorname{TGV}_{\alpha}^{2}(u)<+\infty$. In addition

$$
\|u\|_{B V G_{\alpha}^{2}}=\|u\|_{L^{1}}+\operatorname{TGV}_{\alpha}^{2}(u) \leq\|u\|_{W^{1,1}}+\alpha_{0} \operatorname{TV}^{2}(u) \leq \max \left(1, \alpha_{0}\right)\|u\|_{B H},
$$

which gives the continuity of the embedding mapping.

Corollary 2.1 For any $u \in \mathrm{BH}(\Omega), \mathrm{TV}^{2}(u)=0$ if and only if $u$ is a polynomial function of order 1.

Proof. For any $u \in \mathrm{BH}(\Omega), \mathrm{TV}^{2}(u)=0 \Longrightarrow \mathrm{TGV}_{\alpha}^{2}(u)=0$. Then we use Proposition 3.3 of [15].

The main difference between the two approaches concerns the functions regularity. The $\operatorname{BGV}_{\alpha}^{2}(\Omega)$ functions do not necessarily belong to $\mathrm{L}^{1}(\Omega)$. In particular, the indicator function of smooth open sets belongs to $\operatorname{BGV}_{\alpha}^{2}(\Omega)$ and not to $\mathrm{BH}(\Omega)$. On the other hand, we cannot have Sobolev-type embeddings for $\operatorname{BGV}_{\alpha}^{2}(\Omega)$.

\section{A Second-Order Variational Model for Image Decomposition}

\subsection{Presentation of the Model}

We have already presented a slightly similar model in [11] (see more precisely Remark 4 of [11] where the boundary condition is discussed). Here we provide an existence result that was expected but only proved in the finite dimensional case and give a inf-convolution formulation. We now assume that the image we want to recover $u_{d}$ belongs to $\mathrm{L}^{2}(\Omega)$ and that it can be decomposed as $u_{d}=w+u+v$ where $u, v$ and $w$ are functions that characterize different parts. These components belong to different functional spaces: $v \in \mathrm{BH}(\Omega)$ is the (smooth) second order part, $u$ is a $\operatorname{BV}(\Omega)$ component and $w \in \mathrm{L}^{2}(\Omega)$ is the remainder term. We consider the following cost functional defined on $\mathrm{BV}(\Omega) \times \mathrm{BH}(\Omega)$ :

$$
\mathcal{F}_{\lambda, \mu}(u, v):=\frac{1}{2}\left\|u_{d}-u-v\right\|_{\mathrm{L}^{2}(\Omega)}^{2}+\lambda \mathrm{TV}(u)+\mu \mathrm{TV}^{2}(v),
$$

where $\lambda, \mu>0$. We are looking for a solution to the optimization problem

$$
\inf \left\{\mathcal{F}_{\lambda, \mu}(u, v):(u, v) \in \mathrm{BV}(\Omega) \times \mathrm{BH}_{0}(\Omega)\right\} \quad\left(\mathcal{P}_{\lambda, \mu}\right)
$$

Remark 3.1 We decide to look for the minima of $\mathcal{F}_{\lambda, \mu}$ on BV $(\Omega) \times \mathrm{BH}_{0}(\Omega)$ and not $\mathrm{BV}(\Omega) \times \mathrm{BH}(\Omega)$ to get an existence result. This will cause troubles to set the dual problem because of the computation of Legendre-Fenchel conjugate functions. Nevertheless, the constraint $v \in \mathrm{BH}_{0}(\Omega)$ (that is $\frac{\partial v}{\partial n}=0$ on $\partial \Omega$ ) is a usual one in image processing and the difficulty will be overcome in the discrete setting. 
We expect $v$ to be the smooth colored part of the image, $u$ to be a $\mathrm{BV}(\Omega) \backslash \mathrm{BH}(\Omega)$ function which derivative is a measure supported by the contours and $w:=u_{d}-u-v \in \mathrm{L}^{2}(\Omega)$ is the noise and/or small textures (we shall detail this point later). Problem $\left(\mathcal{P}_{\lambda, \mu}\right)$ can be (formally) viewed as a minimization problem where the regularization term is an inf-convolution. Recall that the inf-convolution has been introduced by J.J. Moreau in [26] (see also [3] p 324). It is defined as

$$
(f \# g)(v):=\inf \{f(u)+g(v-u): u \in V\},
$$

where $f, g: V \rightarrow \mathbb{R} \cup\{+\infty\}$. If we set $V=\mathrm{L}^{2}(\Omega)$,

$$
\Phi_{\lambda}^{1}(u)=\left\{\begin{array}{l}
\lambda \operatorname{TV}(u), \text { if } u \in \operatorname{BV}(\Omega) \\
+\infty, \text { else. }
\end{array} \text { and } \Phi_{\mu}^{2}(v)=\left\{\begin{array}{l}
\mu \operatorname{TV}^{2}(v), \text { if } v \in \mathrm{BH}_{0}(\Omega) \\
+\infty, \text { else. }
\end{array}\right.\right.
$$

then

$\left(\Phi_{\lambda}^{1} \# \Phi_{\mu}^{2}\right)(\varpi)=\inf \left\{\lambda \operatorname{TV}(u)+\mu \operatorname{TV}^{2}(v): u \in \mathrm{BV}(\Omega), \mathrm{v} \in \mathrm{BH}_{0}(\Omega), \mathrm{u}+\mathrm{v}=\varpi\right\}$, and problem $\left(\mathcal{P}_{\lambda, \mu}\right)$ may be written as

$$
\inf \left\{\frac{1}{2}\left\|u_{d}-\varpi\right\|_{\mathrm{L}^{2}(\Omega)}^{2}+\left(\Phi_{\lambda}^{1} \# \Phi_{\mu}^{2}\right)(\varpi): \varpi \in \mathrm{L}^{2}(\Omega)\right\} .
$$

This formulation is to compare to the one by Bredies and al. [15-17]

$$
\inf \left\{\frac{1}{2}\left\|u_{d}-\varpi\right\|_{\mathrm{L}^{2}(\Omega)}^{2}+\mathrm{TGV}_{\alpha}^{2}(\varpi): \varpi \in \mathrm{L}^{2}(\Omega)\right\},
$$

which seems to be more efficient for denoising. However, we are interested in the decomposition components $u$ and $v$. First, we give an existence result for problem $\left(\mathcal{P}_{\lambda, \mu}\right)$.

Theorem 3.1 (Existence) Problem $\left(\mathcal{P}_{\lambda, \mu}\right)$ has at least an optimal solution $\left(u^{*}, v^{*}\right)$ in $\mathrm{BV}(\Omega) \times \mathrm{BH}_{0}(\Omega)$.

Proof. We first prove that the auxiliary problem

$$
\inf \left\{\mathcal{F}_{\lambda, \mu}(u, v):(u, v) \in \mathrm{BV}_{\mathrm{m}}(\Omega) \times \mathrm{BH}_{0}(\Omega)\right\} \quad\left(\mathcal{P}_{\text {aux }}\right)
$$

has an optimal solution. Let $\left(u_{n}, v_{n}\right)_{n \in \mathbb{N}} \in \mathrm{BV}_{\mathrm{m}}(\Omega) \times \mathrm{BH}_{0}(\Omega)$ be a minimizing sequence, i.e.

$$
\lim _{n \rightarrow+\infty} \mathcal{F}_{\lambda, \mu}\left(u_{n}, v_{n}\right)=\inf \left\{\mathcal{F}_{\lambda, \mu}(v):(u, v) \in \mathrm{BV}_{\mathrm{m}}(\Omega) \times \mathrm{BH}_{0}(\Omega)\right\}<+\infty .
$$

Therefore

- $\operatorname{TV}^{2}\left(v_{n}\right)$ is bounded and with Lemma 2.1, $\left\|\nabla v_{n}\right\|_{\mathrm{L}^{1}}$ is bounded as well.

- TV $\left(u_{n}\right)$ is bounded. Using once again Lemma 2.1 yields that $u_{n}$ is bounded in $\mathrm{L}^{1}(\Omega)$. Therefore the sequence $\left(u_{n}\right)_{n \in \mathbb{N}}$ is bounded in $\mathrm{BV}(\Omega)$. 
- As $u_{n}+v_{n}$ is $\mathrm{L}^{2}$-bounded, it is $\mathrm{L}^{1}$-bounded as well so that $v_{n}$ is $\mathrm{L}^{1}$ bounded. As $\left\|\nabla v_{n}\right\|_{\mathrm{L}^{1}}$ and $\mathrm{TV}^{2}\left(v_{n}\right)$ are bounded, this means that the sequence $\left(v_{n}\right)_{n \in \mathbb{N}}$ is bounded in $\operatorname{BH}(\Omega)$.

With the compactness result of Theorem 2.2, this yields that $\left(v_{n}\right)_{n \in \mathbb{N}}$ strongly converges (up to a subsequence) in $\mathrm{W}^{1,1}(\Omega)$ to $v^{*} \in \mathrm{BH}(\Omega)$. In addition, as the normal derivative operator is continuous (as mentionned before), then $v^{*} \in \mathrm{BH}_{0}(\Omega)$. Similarly $\left(u_{n}\right)_{n \in \mathbb{N}}$ strongly converges (up to a subsequence) in $\mathrm{L}^{1}(\Omega)$ to $u^{*} \in \mathrm{BV}_{\mathrm{m}}(\Omega)$. Moreover $u_{n}+v_{n}$ weakly converges to $u^{*}+v^{*}$ in $\mathrm{L}^{2}(\Omega)$. With Theorem 2.1 we get

$$
\mathrm{TV}\left(u^{*}\right) \leq \liminf _{n \rightarrow+\infty} \mathrm{TV}\left(u_{n}\right), \mathrm{TV}^{2}\left(v^{*}\right) \leq \liminf _{n \rightarrow+\infty} \mathrm{TV}^{2}\left(v_{n}\right) .
$$

So, $\mathcal{F}_{\lambda, \mu}\left(u^{*}, v^{*}\right) \leq \liminf _{n \rightarrow+\infty} \mathcal{F}_{\lambda, \mu}\left(u_{n}, v_{n}\right)=\min \left(\mathcal{P}_{\text {aux }}\right)$ and $\left(u^{*}, v^{*}\right)$ is a solution to $\left(\mathcal{P}_{\text {aux }}\right)$.

For every $(u, v) \in \mathrm{BV}(\Omega) \times \mathrm{BH}_{0}(\Omega),\left(u-m_{u}, v+m_{u}\right) \in \mathrm{BV}_{\mathrm{m}}(\Omega) \times \mathrm{BH}_{0}(\Omega)$ where $m_{u}=\frac{1}{|\Omega|} \int_{\Omega} u$ is the mean value of $u$. Moreover

$$
\mathcal{F}_{\lambda, \mu}(u, v)=\mathcal{F}_{\lambda, \mu}\left(u-m_{u}, v+m_{u}\right) \geq \mathcal{F}_{\lambda, \mu}\left(u^{*}, v^{*}\right) .
$$

Therefore $\left(u^{*}, v^{*}\right)$ is an optimal solution to $\left(\mathcal{P}_{\lambda, \mu}\right)$.

Remark 3.2 Uniqueness of the solution is challenging. We shall prove partial results in section 4.2 .

\subsection{Optimality Conditions}

In what follows, we set $\mathcal{N}(u):=\frac{1}{2}\|u\|_{2}^{2}$ for any $u \in \mathrm{L}^{2}(\Omega)$ and fix $\lambda>0, \mu>0$. From now on, $(\bar{u}, \bar{v})$ denotes any solution $\left(\mathcal{P}_{\lambda, \mu}\right)$. It is characterized by

$$
\begin{aligned}
& \bar{u}=\operatorname{argmin}\left\{\frac{1}{2}\left\|u_{d}-\bar{v}-u\right\|^{2}+\Phi_{\lambda}^{1}(u), u \in \mathrm{L}^{2}(\Omega)\right\}, \\
& \bar{v}=\operatorname{argmin}\left\{\frac{1}{2}\left\|u_{d}-\bar{u}-v\right\|^{2}+\Phi_{\mu}^{2}(v), v \in \mathrm{L}^{2}(\Omega)\right\}
\end{aligned}
$$

where $\Phi_{\lambda}^{1}$ and $\Phi_{\mu}^{2}$ have been defined in section 3.1. We may derive optimality conditions in a standard way :

Theorem $3.2(\bar{u}, \bar{v})$ is a solution to $\left(\mathcal{P}_{\lambda, \mu}\right)$ if and only if

$$
\bar{w}:=u_{d}-\bar{u}-\bar{v} \in \partial \Phi_{\lambda}^{1}(\bar{u}) \cap \partial \Phi_{\mu}^{2}(\bar{v}) .
$$

The proof is obvious. The notation $\langle\cdot, \cdot\rangle$ stands for the duality product between $V^{\prime}$ et $V$ and $\partial f(u)$ denotes the subdifferential of $f$ at $u$ where $f: V \rightarrow \mathbb{R}$ :

$$
\partial f(u):=\left\{u^{*} \in V^{\prime}: \forall v \in V \quad f(v)-f(u) \geq\left\langle u^{*}, v-u\right\rangle\right\} .
$$




\subsection{Inf-Convolution Formulation}

We have already noticed that the penalization term in $\left(\mathcal{P}_{\lambda, \mu}\right)$ is an infimal convolution term. In addition, $\left(\mathcal{P}_{\lambda, \mu}\right)$ can be viewed as successive inf-convolution processes.

Lemma 3.1 The functionals $\mathcal{N} \# \Phi_{\lambda}^{1}$ and $\mathcal{N} \# \Phi_{\mu}^{2}$ are convex and continuous from $\mathrm{L}^{2}(\Omega)$ to $\mathrm{L}^{2}(\Omega)$.

Proof. In the sequel we set $\Phi=\Phi_{\lambda}^{1}$ or $\Phi_{\mu}^{2}$ indifferently. As $\Phi$ and $\mathcal{N}$ are convex so is $\mathcal{N} \# \Phi$ (see [27] for example). Let be $u \in \mathrm{L}^{2}(\Omega)$ :

$$
(\mathcal{N} \# \Phi)(u)=\inf \left\{\frac{1}{2}\|u-v\|_{2}^{2}+\Phi(v): v \in \mathrm{L}^{2}(\Omega)\right\} \leq \frac{1}{2}\|u\|_{2}^{2}+\Phi(0)=\frac{1}{2}\|u\|_{2}^{2} .
$$

As $(\mathcal{N} \# \Phi)(0)=0$ this gives the $\mathcal{N} \# \Phi$ continuity at 0 and its boundedness in a neighborhood of 0 . As it is convex, it is continuous on its whole domain $\mathrm{L}^{2}(\Omega)$ (see [28] for example).

Note that problem (10) is equivalent to the fact that $\bar{u}$ realizes the infimum in the definition of $\mathcal{N} \# \Phi_{\lambda}^{1}\left(u_{d}-\bar{v}\right)$ and (11) means that $\bar{v}$ realizes the infimum in $\mathcal{N} \# \Phi_{\mu}^{2}\left(u_{d}-\bar{u}\right)$. In fact, problem $\left(\mathcal{P}_{\lambda, \mu}\right)$ can be written as successive infconvolution processes. More precisely we have:

Theorem 3.3 Let $(\bar{u}, \bar{v}) \in \mathrm{BV}(\Omega) \times \mathrm{BH}_{0}(\Omega)$ be a solution to $\left(\mathcal{P}_{\lambda, \mu}\right)$. Then

$$
\begin{aligned}
\bar{m} & =\mathcal{N}\left(u_{d}-\bar{u}-\bar{v}\right)+\Phi_{\lambda}^{1}(\bar{u})+\Phi_{\mu}^{2}(\bar{v}) \\
& \left.\left.=\left(\mathcal{N} \# \Phi_{\lambda}^{1}\right)\right)\left(u_{d}-\bar{v}\right)+\Phi_{\mu}^{2}(\bar{v})=\left(\mathcal{N} \# \Phi_{\mu}^{2}\right)\right)\left(u_{d}-\bar{u}\right)+\Phi_{\lambda}^{1}(\bar{u}) \\
& =\left(\Phi_{\lambda}^{1} \#\left(\mathcal{N} \# \Phi_{\mu}^{2}\right)\right)\left(u_{d}\right)=\left(\Phi_{\mu}^{2} \#\left(\mathcal{N} \# \Phi_{\lambda}^{1}\right)\right)\left(u_{d}\right) .
\end{aligned}
$$

where $\bar{m}:=\inf \left(\mathcal{P}_{\lambda, \mu}\right)$.

Proof. Let $(\bar{u}, \bar{v}) \in \mathrm{BV}(\Omega) \times \mathrm{BH}_{0}(\Omega)$ be a solution to $\left(\mathcal{P}_{\lambda, \mu}\right)$.

Then, for every $(u, v) \in \mathrm{BV}(\Omega) \times \mathrm{BH}_{0}(\Omega)$, we get

$$
\bar{m}=\mathcal{N}\left(u_{d}-\bar{u}-\bar{v}\right)+\Phi_{\lambda}^{1}(\bar{u})+\Phi_{\mu}^{2}(\bar{v}) \leq \mathcal{N}\left(u_{d}-u-v\right)+\Phi_{\lambda}^{1}(\bar{u})+\Phi_{\mu}^{2}(v) .
$$

This gives, for every $v \in \mathrm{BH}_{0}(\Omega)$

$$
\bar{m} \leq \inf _{u \in \mathrm{L}^{2}(\Omega)} \mathcal{N}\left(u_{d}-u-v\right)+\Phi_{\lambda}^{1}(\bar{u})+\Phi_{\mu}^{2}(v)=\left(\mathcal{N} \# \Phi_{\lambda}^{1}\right)\left(u_{d}-v\right)+\Phi_{\mu}^{2}(v)
$$

so that

$$
\bar{m} \leq \inf _{v \in \mathrm{BH}_{0}(\Omega)}\left(\mathcal{N} \# \Phi_{\lambda}^{1}\right)\left(u_{d}-v\right)+\Phi_{\mu}^{2}(v) \leq\left(\Phi_{\mu}^{2} \#\left(\mathcal{N} \# \Phi_{\lambda}^{1}\right)\right)\left(u_{d}\right) .
$$

Similarly

$$
\bar{m} \leq\left(\Phi_{\lambda}^{1} \#\left(\mathcal{N} \# \Phi_{\mu}^{2}\right)\right)\left(u_{d}\right)
$$

Conversely, we get for every $(u, v) \in \mathrm{BV}(\Omega) \times \mathrm{BH}_{0}(\Omega)$ by definition of infconvolution:

$\left(\Phi_{\mu}^{2} \#\left(\mathcal{N} \# \Phi_{\lambda}^{1}\right)\right)\left(u_{d}\right) \leq\left(\mathcal{N} \# \Phi_{\lambda}^{1}\right)\left(u_{d}-v\right)+\Phi_{\mu}^{2}(v) \leq \mathcal{N}\left(u_{d}-v-u\right)+\Phi_{\lambda}^{1}(u)+\Phi_{\mu}^{2}(v)$, so that $\left(\Phi_{\mu}^{2} \#\left(\mathcal{N} \# \Phi_{\lambda}^{1}\right)\right)\left(u_{d}\right) \leq \bar{m}$.

We finally obtain $\bar{m}=\left(\Phi_{\lambda}^{1} \#\left(\mathcal{N} \# \Phi_{\mu}^{2}\right)\right)\left(u_{d}\right)=\left(\Phi_{\mu}^{2} \#\left(\mathcal{N} \# \Phi_{\lambda}^{1}\right)\right)\left(u_{d}\right)$. 
3.4 Computing the Legendre-Fenchel Conjugate Functions

We are going to write the dual problem of $\left(\mathcal{P}_{\lambda, \mu}\right)$ and need to compute the conjugate functions of $\Phi_{\lambda}^{1}, \Phi_{\mu}^{2}$ and $\tilde{f}: u \mapsto f\left(u_{d}+u\right)$. We recall that, if $f: V \rightarrow \mathbb{R} \cup\{+\infty\}$, the Legendre-Fenchel conjugate $f^{*}$ is defined on $V^{\prime}$ as

$$
\forall u^{*} \in V^{\prime} \quad f^{*}\left(u^{*}\right):=\sup _{u \in V}\left\langle u^{*}, u\right\rangle-f(u) .
$$

We obviously have $(\lambda f)^{*}\left(u^{*}\right)=\lambda f^{*}\left(\frac{u^{*}}{\lambda}\right)$, for every $\lambda>0$ and $u^{*} \in V^{\prime}$ and the following useful result as well [3]:

Proposition 3.1 Let $V$ be a normed space and $f: V \rightarrow \mathbb{R} \cup\{+\infty\}$ a closed and convex, proper function. then

$$
u^{*} \in \partial f(u) \Longleftrightarrow u \in \partial f^{*}\left(u^{*}\right) \Longleftrightarrow f(u)+f^{*}\left(u^{*}\right)=\left\langle u^{*}, u\right\rangle,
$$

where $\langle\cdot, \cdot\rangle$ denotes the duality $V^{\prime}-V$ product.

Lemma 3.2 Let be $f: \mathrm{L}^{2}(\Omega) \rightarrow \mathbb{R} \cup\{+\infty\}$ and $\tilde{f}$ such that $\tilde{f}(u)=f\left(u_{d}+u\right)$. Then the Legendre-Fenchel conjugate function of $\tilde{f}$ writes

$$
\forall u^{*} \in \mathrm{L}^{2}(\Omega) \quad(\tilde{f})^{*}\left(u^{*}\right)=f^{*}\left(u^{*}\right)-\left(u^{*}, u_{d}\right)_{2},
$$

where $(\cdot, \cdot)_{2}$ denotes the $\mathrm{L}^{2}(\Omega)$ inner product.

Proof. Let be $u^{*} \in \mathrm{L}^{2}(\Omega)$. We have

$$
\begin{aligned}
(\tilde{f})^{*}\left(u^{*}\right) & =\sup _{u \in \mathrm{L}^{2}(\Omega)}\left(u, u^{*}\right)_{2}-f\left(u_{d}+u\right)=\sup _{w \in \mathrm{L}^{2}(\Omega)}\left(w-u_{d}, u^{*}\right)_{2}-f(w) \\
& =\sup _{w \in \mathrm{L}^{2}(\Omega)}\left(w, u^{*}\right)_{2}-f(w)-\left(u_{d}, u^{*}\right)_{2}=f^{*}\left(u^{*}\right)-\left(u^{*}, u_{d}\right)_{2} .
\end{aligned}
$$

In the sequel $\mathbf{1}_{C}$ denotes the indicator function of a set $C$ :

$$
\mathbf{1}_{C}(u):=\left\{\begin{array}{l}
0, \text { if } u \in C \\
+\infty, \text { else }
\end{array}\right.
$$

Lemma 3.3 The conjugate function of $\Phi_{\lambda}^{1}$ is $\left(\Phi_{\lambda}^{1}\right)^{*}=\lambda \mathbf{1}_{\lambda \mathcal{K}_{1}}$, where $\mathcal{K}_{1}$ is the $\mathrm{L}^{2}$-closure of

$$
\mathbf{K}_{1}:=\left\{\xi=\operatorname{div} \varphi: \varphi \in \mathcal{C}_{c}^{1}(\Omega),\|\varphi\|_{\infty} \leq 1\right\} .
$$

The conjugate function of $\Phi_{\mu}^{2}$ is $\left(\Phi_{\mu}^{2}\right)^{*}=\mu \mathbf{1}_{\mu \mathcal{K}_{2}}$, where $\mathcal{K}_{2} \supset \operatorname{cl}\left(\mathbf{K}_{2}\right)$ and $\mathrm{cl}\left(\mathbf{K}_{2}\right)$ is the $\mathrm{L}^{2}$-closure of

$$
\mathbf{K}_{2}:=\left\{\xi=\operatorname{div}^{2} \psi: \psi \in \mathcal{C}_{c}^{2}\left(\Omega, \mathbb{R}^{d \times d}\right),\|\psi\|_{\infty} \leq 1\right\}
$$


Proof. It is known that the conjugate $\mathrm{TV}^{*}$ of $\mathrm{TV}$ is the indicator function of $\mathcal{K}_{1}$ (see $[23,29]$ for example). As $\Phi_{\lambda}^{1}=\lambda \mathrm{TV}$ (or $+\infty$ outside $B V(\Omega)$ ), then $\left(\Phi_{\lambda}^{1}\right)^{*}\left(u^{*}\right)=\lambda \mathrm{TV}^{*}\left(\frac{u^{*}}{\lambda}\right)$. This gives the result.

The second result is not exactly the same, since $\Phi_{\mu}^{2}$ is equal to $\mu \mathrm{TV}^{2}$ on $\mathrm{BH}_{0}(\Omega)$ and $+\infty$ outside (and in particular on $\mathrm{BH}(\Omega) \backslash \mathrm{BH}_{0}(\Omega)$ ). Therefore the conjugate of $\Phi_{\mu}^{2}$ is not the same as the conjugate of $\mu \mathrm{TV}^{2}$. We know that the conjugate function of $\mathrm{TV}^{2}$ is $\mathbf{1}_{c l\left(\mathbf{K}_{2}\right)}$ (see [12]); as $\Phi_{1}^{2}$ is positively homogeneous $(\mu=1)$, it is the indicator function of a closed subset $\mathcal{K}_{2}$ of $\mathrm{L}^{2}(\Omega)$. Moreover

$$
\begin{aligned}
& \mathbf{1}_{\mathcal{K}_{2}}\left(v^{*}\right)=\left(\Phi_{1}^{2}\right)^{*}\left(v^{*}\right)=\sup _{v \in \mathrm{BH}_{0}}\left\langle v^{*}, v\right\rangle-\mathrm{TV}^{2}(v) \\
& \leq \sup _{v \in \mathrm{BH}}\left\langle v^{*}, v\right\rangle-\mathrm{TV}^{2}(v)=\mathbf{1}_{c l\left(\mathbf{K}_{2}\right)}\left(v^{*}\right) .
\end{aligned}
$$

This implies that $\operatorname{cl}\left(\mathbf{K}_{2}\right) \subset \mathcal{K}_{2}$ but we cannot prove the converse inclusion that would provide a result similar to the first order case. We end the proof with the same argument as in the BV case.

Eventually it is easy to see that $\mathcal{N}^{*}=\mathcal{N}$.

\subsection{Dual Problem to $\left(\mathcal{P}_{\lambda, \mu}\right)$}

In the present subsection, we shall use convex duality tools that we recall thereafter (see [3] for example).

Theorem 3.4 ([3] p. 366) Let $V$ be a Banach space, $f, g: V \rightarrow \mathbb{R} \cup\{+\infty\}$ lower semi-continuous convex functions and $A$ a linear continuous operator from $V$ to $V$. Assume there exists $u_{o} \in \operatorname{dom} g$, and that $f$ is continuous at $A u_{o}$. Then

$$
\inf _{u \in V}(f(A u)+g(u))=\max _{u^{*} \in V^{\prime}}\left(-f^{*}\left(u^{*}\right)-g^{*}\left(-A^{*} u^{*}\right)\right) .
$$

Moreover, if $\bar{u}$ is a solution to the primal problem and $\bar{u}^{*}$ is a solution to the dual one, then

$$
\bar{u}^{*} \in \partial f(A \bar{u}) \text { and }-A^{*} \bar{u}^{*} \in \partial g(\bar{u}),
$$

where $\partial f(u)$ stands for the subdifferential of $f$ at $u$.

Theorem 3.5 ([3] p. 328) Let $V$ be a Banach space and $f, g: V \rightarrow \mathbb{R} \cup\{+\infty\}$ proper functions. Then $(f \# g)^{*}=f^{*}+g^{*}$. In addition if $f$ and $g$ satisfy the assumptions of Theorem 3.4, then $(f+g)^{*}=f^{*} \# g^{*}$.

Now we may compute the dual problem to $\left(\mathcal{P}_{\lambda, \mu}\right)$ and get the following:

Theorem 3.6 The dual problem to $\left(\mathcal{P}_{\lambda, \mu}\right)$ writes

$$
\inf \left\{\frac{1}{2}\left\|u_{d}-w\right\|_{2}^{2}: w \in \lambda \mathcal{K}_{1} \cap \mu \mathcal{K}_{2}\right\} .
$$

The unique solution $w^{*}$ is the $\mathrm{L}^{2}$-projection of $u_{d}$ on the closed and convex set $\lambda \mathcal{K}_{1} \cap \mu \mathcal{K}_{2}$. 
Proof. Solving problem $\left(\mathcal{P}_{\lambda, \mu}\right)$ is equivalent to solving

$$
\inf _{u \in \mathrm{L}^{2}(\Omega)}\left(\mathcal{N} \# \Phi_{\mu}^{2}\right)\left(u_{d}-u\right)+\Phi_{\lambda}^{1}(u)=\inf _{u \in \mathrm{L}^{2}(\Omega)}\left(\widetilde{\mathcal{N} \# \Phi_{\mu}^{2}}\right)(A u)+\Phi_{\lambda}^{1}(u)
$$

where $\left(\widetilde{\mathcal{N} \# \Phi_{\mu}^{2}}\right)(w)=\left(\mathcal{N} \# \Phi_{\mu}^{2}\right)\left(u_{d}+w\right)$ and $A u=-u$.

It is clear that $A^{*}=A$. Moreover, $\Phi_{\lambda}^{1}$ is lsc with respect to the $\mathrm{L}^{1}$ - topology and $\mathrm{L}^{2}$ - topology since $\Omega$ is bounded. As $\mathcal{N} \# \Phi_{\mu}^{2}, \widetilde{\mathcal{N} \# \Phi_{\mu}^{2}}, A$ and $\Phi_{\lambda}^{1}$ fulfill assumptions of Theorem 3.4, the dual problem of $\left(\mathcal{P}_{\lambda, \mu}\right)$ writes

$$
\max \left\{-\left(\widetilde{\mathcal{N} \# \Phi_{\mu}^{2}}\right)^{*}(w)-\left(\Phi_{\lambda}^{1}\right)^{*}(w): w \in \mathrm{L}^{2}(\Omega)\right\} . \quad \quad\left(\mathcal{P}_{\lambda, \mu}^{*}\right)
$$

Using Lemma 3.2 and Theorem 3.5, it is easy to see that

$$
\begin{aligned}
\left(\widetilde{\mathcal{N} \# \Phi_{\mu}^{2}}\right)^{*}(w) & =-\left(u_{d}, w\right)_{2}+\left(\mathcal{N} \# \Phi_{\mu}^{2}\right)^{*}(w)=-\left(u_{d}, w\right)_{2}+\mathcal{N}^{*}(w)+\left(\Phi_{\mu}^{2}\right)^{*}(w) \\
& =-\left(u_{d}, w\right)_{2}+\mathcal{N}(w)+\left(\Phi_{\mu}^{2}\right)^{*}(w) .
\end{aligned}
$$

Therefore, $\left(\mathcal{P}_{\lambda, \mu}^{*}\right)$ writes

$$
\begin{gathered}
\max _{w \in \mathrm{L}^{2}(\Omega)}\left(u_{d}, w\right)_{2}-\mathcal{N}(w)-\left(\Phi_{\lambda}^{1}\right)^{*}(w)-\left(\Phi_{\mu}^{2}\right)^{*}(w), \\
\Longleftrightarrow-\min _{w \in \mathrm{L}^{2}(\Omega)}-\left(u_{d}, w\right)_{2}+\mathcal{N}(w)+\left(\Phi_{\lambda}^{1}\right)^{*}(w)+\left(\Phi_{\mu}^{2}\right)^{*}(w) .
\end{gathered}
$$

Finally, $\left(\mathcal{P}_{\lambda, \mu}^{*}\right)$ is equivalent to

$$
\min \left\{\frac{1}{2}\left\|u_{d}-w\right\|_{2}^{2}: w \in \lambda \mathcal{K}_{1} \cap \mu \mathcal{K}_{2}\right\} .
$$

The dual problem has obviously a unique solution $w^{*}$ which is the $\mathrm{L}^{2}$-projection of $u_{d}$ on the closed and convex set $\lambda \mathcal{K}_{1} \cap \mu \mathcal{K}_{2}$.

In the sequel we denote $\Pi_{\mathcal{K}}$ the $\mathrm{L}^{2}$-projection on a closed and convex set $\mathcal{K}$. Next, we have a relation between the solutions to $\left(\mathcal{P}_{\lambda, \mu}\right)$ and the (unique) solution of the dual problem.

Theorem 3.7 1. Let $w^{*}$ be the (unique) solution to the dual problem $\left(\mathcal{P}_{\lambda, \mu}^{*}\right)$, namely $w^{*}=\Pi_{\lambda \mathcal{K}_{1} \cap \mu \mathcal{K}_{2}}\left(u_{d}\right)$. Then, there exists $(\bar{u}, \bar{v}) \in \mathrm{BV}(\Omega) \times \mathrm{BH}_{0}(\Omega)$ an optimal solution to $\left(\mathcal{P}_{\lambda, \mu}\right)$ such that

$$
w^{*}=u_{d}-\bar{u}-\bar{v} \text { and } w^{*} \in \partial \Phi_{\mu}^{2}(\bar{v}) \cap \partial \Phi_{\lambda}^{1}(\bar{u}) .
$$

2. Conversely, if $(\bar{u}, \bar{v}) \in \mathrm{BV}(\Omega) \times \mathrm{BH}_{0}(\Omega)$ is any solution to $\left(\mathcal{P}_{\lambda, \mu}\right)$, then

$$
\bar{w}=u_{d}-\bar{u}-\bar{v}=\Pi_{\lambda \mathcal{K}_{1} \cap \mu \mathcal{K}_{2}}\left(u_{d}\right) .
$$


Proof. Let $(\bar{u}, \tilde{v})$ be a solution to $\left(\mathcal{P}_{\lambda, \mu}\right)$. A direct consequence of Theorem 3.4 is $w^{*} \in \partial \Phi_{\lambda}^{1}(\bar{u})$ and $w^{*} \in \partial\left(\widetilde{\mathcal{N} \# \Phi_{\mu}^{2}}\right)(-\bar{u})$. A simple calculus shows that

$$
\partial\left(\widetilde{\mathcal{N} \# \Phi_{\mu}^{2}}\right)(-\bar{u})=\partial\left(\mathcal{N} \# \Phi_{\mu}^{2}\right)\left(u_{d}-\bar{u}\right),
$$

so that

$$
w^{*} \in \partial \Phi_{\lambda}^{1}(\bar{u}) \cap \partial\left(\mathcal{N} \# \Phi_{\mu}^{2}\right)\left(u_{d}-\bar{u}\right) .
$$

As $\left(\mathcal{N} \# \Phi_{\mu}^{2}\right)\left(u_{d}-\bar{u}\right)=\mathcal{N}\left(u_{d}-\bar{u}-\tilde{v}\right)+\Phi_{\mu}^{2}(\tilde{v})$

$$
=\operatorname{argmin}\left\{\frac{1}{2}\left\|v+\bar{u}-u_{d}\right\|^{2}+\Phi_{\mu}^{2}(v): v \in \mathrm{L}^{2}(\Omega)\right\},
$$

then $u_{d}-\tilde{v}-\bar{u} \in \partial \Phi_{\mu}^{2}(\tilde{v})$; so, the inf-convolution is exact and we get [27]

$$
\partial\left(\mathcal{N} \# \Phi_{\mu}^{2}\right)\left(u_{d}-\bar{u}\right)=\bigcup_{v \in \mathrm{L}^{2}(\Omega)} \partial \mathcal{N}\left(u_{d}-\bar{u}-v\right) \cap \partial \Phi_{\mu}^{2}(v) .
$$

As $\partial \mathcal{N}\left(u_{d}-\bar{u}-v\right)=\left\{u_{d}-\bar{u}-v\right\}$, there exists $\bar{v} \in \mathrm{L}^{2}(\Omega)$ such that $w^{*}=u_{d}-\bar{u}-\bar{v} \in \partial \Phi_{\mu}^{2}(\bar{v})$. So,

$$
w^{*} \in \partial \Phi_{\mu}^{2}(\bar{v}) \cap \partial \Phi_{\lambda}^{1}(\bar{u}),
$$

with $\bar{v}=u_{d}-\bar{u}-w^{*}$. This proves that $(\bar{u}, \bar{v})$ is a solution to $\left(\mathcal{P}_{\lambda, \mu}\right)$ as well: we use Theorem 3.2 with $\bar{w}=w^{*}$ to conclude.

Let us prove the converse property: let $(\bar{u}, \bar{v}) \in \mathrm{BV}(\Omega) \times \mathrm{BH}_{0}(\Omega)$ be a solution to $\left(\mathcal{P}_{\lambda, \mu}\right)$ and $\bar{w}=u_{d}-\bar{u}-\bar{v}$. Theorem 3.2 gives $\bar{w} \in \partial \Phi_{\lambda}^{1}(\bar{u}) \cap \partial \Phi_{\mu}^{2}(\bar{v})$, that is

$$
\bar{u} \in \partial\left(\Phi_{\lambda}^{1}\right)^{*}(\bar{w}) \text { and } \bar{v} \in \partial\left(\Phi_{\mu}^{2}\right)^{*}(\bar{w}) .
$$

With the previous computations, we get $\bar{u} \in \partial \lambda \mathbf{1}_{\lambda \mathcal{K}_{1}}(\bar{w})$ and $\bar{v} \in \partial \lambda \mathbf{1}_{\mu \mathcal{K}_{2}}(\bar{w})$. Therefore

$$
\forall w \in \lambda \mathcal{K}_{1} \cap \mu \mathcal{K}_{2} \quad\langle\bar{u}, w-\bar{w}\rangle \leq 0 \text { and }\langle\bar{v}, w-\bar{w}\rangle \leq 0 .
$$

Adding the above inequalities gives

$$
\forall w \in \lambda \mathcal{K}_{1} \cap \mu \mathcal{K}_{2} \quad\langle\bar{u}+\bar{v}, w-\bar{w}\rangle=\left\langle u_{d}-\bar{w}, w-\bar{w}\right\rangle \leq 0 .
$$

This is equivalent to (16).

Corollary 3.1 If $(\bar{u}, \bar{v})$ is a solution to $\left(\mathcal{P}_{\lambda, \mu}\right)$, then $\bar{w}=\bar{u}+\bar{v}$ is unique. In particular, there is a unique solution to $\left(\mathcal{P}_{\lambda, \mu}\right)$ such that $\bar{u}=0$ almost everywhere.

Remark 3.3 We cannot permute the roles of $\Phi_{\lambda}^{1}$ and $\Phi_{\mu}^{2}$ in the previous proof because $\Phi_{\mu}^{2}$ is not lower semi-continuous with respect to the $\mathrm{L}^{2}$-topology. Indeed $\mathrm{L}^{2}(\Omega)$ is not embedded in $\mathrm{W}^{1,1}(\Omega)$. 


\section{Solution Properties $(d \leq 2)$}

\subsection{Structure of the Solutions}

Recall $[5,21]$ that the Meyer space $\mathrm{G}(\Omega)$ is defined as

$\mathrm{G}(\Omega):=\left\{f \in \mathrm{L}^{2}(\Omega): \exists \varphi=\left(\varphi_{1}, \varphi_{2}\right) \in \mathrm{L}^{\infty}\left(\Omega, \mathbb{R}^{2}\right), f=\operatorname{div} \varphi\right.$ and $\varphi \cdot n=0$ on $\left.\partial \Omega\right\}$,

where $n$ is the outer normal vector to $\partial \Omega$. This space is endowed with a norm denoted by $\|\cdot\|_{G}$ and defined as

$$
\|f\|_{G}:=\inf \left\{\left\|\sqrt{\varphi_{1}^{2}+\varphi_{2}^{2}}\right\|_{\infty}: f=\operatorname{div} \varphi, \varphi \cdot n=0 \text { on } \partial \Omega\right\} .
$$

We shall need the

Lemma 4.1 ( [21] or [5], Lemma 2.1) For every $u \in \mathrm{BV}(\Omega)$ and $g \in \mathrm{G}(\Omega)$ then

$$
\left|\int_{\Omega} u(x) g(x) d x\right| \leq \mathrm{TV}(u)\|g\|_{G} .
$$

We may now precise the structure of a generic solution.

Theorem 4.1 Let $(\bar{u}, \bar{v}) \in \mathrm{BV}(\Omega) \times \mathrm{BH}_{0}(\Omega)$ be a solution to problem $\left(\mathcal{P}_{\lambda, \mu}\right)$ (for fixed $\lambda$ and $\mu$ ) and set $\bar{w}=u_{d}-\bar{u}-\bar{v}$. Then,

i. $\bar{w}=u_{d}-\bar{u}-\bar{v} \in \mathrm{G}(\Omega)$

ii. If $d=2$ and $\Omega$ satisfies assumption (1), $\bar{v}$ is continuous on $\bar{\Omega}$.

iii. If $d=2, \Omega$ satisfies assumption (1) and $u_{d} \in \operatorname{BV}(\Omega) \cap \mathrm{L}^{\infty}(\Omega)$, then the jump set of $\bar{u}$ is included in the jump set of $u_{d}$.

Proof. (i) This is a direct consequence of Theorem 3.7. Indeed $\bar{w} \in \lambda \mathcal{K}_{1}$. Therefore, there exists a sequence $\varphi_{n} \in \mathcal{C}_{c}^{1}\left(\Omega, \mathbb{R}^{2}\right)$ with $\left\|\varphi_{n}\right\|_{\infty} \leq 1$ such that $w_{n}=\lambda \operatorname{div}\left(\varphi_{n}\right) \mathrm{L}^{2}$-converges to $\bar{w}$. As $\left\|\varphi_{n}\right\|_{\infty} \leq 1$, on may extract a weak-star subsequence that converges to $\bar{\varphi}$ in $\mathrm{L}^{\infty}(\Omega)$. Therefore, $\bar{\varphi} \in \mathrm{L}^{\infty}(\Omega)$ and $\bar{\varphi} \cdot n=0$ on $\partial \Omega$. So, we get :

$$
\forall u \in \mathcal{D}(\Omega) \quad\left(w_{n}, u\right)_{\mathrm{L}^{2}}=\lambda \int_{\Omega} \operatorname{div} \varphi_{n} u=-\lambda \int_{\Omega} \varphi_{n} \nabla u \rightarrow-\lambda \int_{\Omega} \bar{\varphi} \nabla u .
$$

As $\left(w_{n}, u\right)_{\mathrm{L}^{2}} \rightarrow(\bar{w}, u)_{\mathrm{L}^{2}}$ this gives

$$
(\bar{w}, u)=-\lambda\langle\bar{\varphi}, \nabla u\rangle=\lambda\langle\operatorname{div} \bar{\varphi}, u\rangle,
$$

in the distributional sense. Therefore, $\bar{w}=\operatorname{div}(\lambda \bar{\varphi})$. Moreover, $\bar{\varphi} \cdot n=0$ on $\partial \Omega$ since $\varphi_{n}$ has compact support. This proves that $\bar{w} \in \mathrm{G}(\Omega)$.

(ii) Assumption (1) implies that $\bar{v} \in \mathrm{BH}(\Omega)$ is continuous (Theorem 2.2, (iii)). (iii) With (ii), the jump discontinuity set of $u_{d}$ is the same as the one of $u_{d}-\bar{v}$. Moreover $\bar{u}$ is a solution to

$$
\min \left\{\frac{1}{2}\left\|u_{d}-\bar{v}-u\right\|^{2}+\lambda \operatorname{TV}(u): u \in \operatorname{BV}(\Omega)\right\} .
$$

Therefore, following [30], Theorem 3.3, we get the result. 
Remark 4.1 - The point (i) means that $\bar{w}$ is an oscillating function: this is consistent with the fact that we expect $\bar{w}$ to be the noise and/or micro-textures. - The continuity of $\bar{v}$ still holds if $d \geq 2$. Assumptions on $\Omega$ are slightly different (see $[13,25])$.

- Any variational model

$$
\min \left\{\frac{1}{2}\left\|u_{d}-u-v\right\|_{\mathrm{L}^{2}(\Omega)}^{2}+\lambda \mathrm{TV}(u)+\Phi(v): u \in \mathrm{BV}(\Omega), \mathrm{v} \in \mathcal{V}\right\},
$$

would satisfy the result of iii) (with a functional $\Phi$ such that a solution $(\bar{u}, \bar{v}$ ) exists), if $\mathcal{V}$ contains $\mathcal{C}^{0}(\bar{\Omega})$ (which is the case if $\mathcal{V}=\mathrm{BH}(\Omega)$ ). Indeed, the result of [30] applies as soon as $\bar{u}$ is the solution of a TV-type problem.

- Since $\left.\bar{v} \in \mathrm{W}^{1,1}(\Omega) \cap \mathrm{L}^{\infty}(\Omega)\right)$, if we assume $u_{d} \in \operatorname{SBV}(\Omega) \cap \mathrm{L}^{\infty}(\Omega)$ then $u_{d}+\bar{v} \in \operatorname{SBV}(\Omega)$. Here $\operatorname{SBV}(\Omega)$ is the space of special bounded variation functions $[3,4]$. Of course, the jump set of $\bar{u}$ is included in the jump set of $u_{d}$, but we cannot prove that the non absolutely continuous part of the derivative of $\bar{u}$ is concentrated on its jump set. So, we cannot prove that $\bar{u}$ belongs to $\operatorname{SBV}(\Omega)$ though the numerical simulations of [31] allow to think it is the case ( $\bar{u}$ is piecewise constant).

Corollary 4.1 Let $(\bar{u}, \bar{v}) \in \mathrm{BV}(\Omega) \times \mathrm{BH}_{0}(\Omega)$ be a solution to problem $\left(\mathcal{P}_{\lambda, \mu}\right)$ (for fixed $\lambda$ and $\mu$ ) and set $\bar{w}=u_{d}-\bar{u}-\bar{v}$. Then $\int_{\Omega} \bar{w}(x) d x=0$.

Proof. This is a direct consequence of Proposition 2.1 of [5] that gives

$$
\mathrm{G}(\Omega)=\left\{g \in \mathrm{L}^{2}(\Omega): \int_{\Omega} g(x) d x=0\right\}
$$

and that $\bar{w} \in \mathrm{G}(\Omega)$.

The previous theorem holds if $u_{d} \in \mathrm{BV}(\Omega)$. This is not the case if $u_{d}$ is noisy, for example. In the case where $u_{d} \notin \mathrm{BV}(\Omega)$, we have the following results due to W. Ring [32]. We first consider the $1 \mathrm{D}$ case where $\Omega=] a, b[$. Following Proposition 4 of [32], if we assume that

$\forall \mathcal{U}$ open subset of $] a, b[$ with positive Lebesgue measure $u_{d}$ does not coincide on $\mathcal{U}$ with some function $u \in \mathrm{BV}(] a, b[)$,

then $u_{d}-\bar{v}$ satisfies $\left(\mathcal{H}_{1}\right)$ and we get $\mathrm{D}_{a} \bar{u}=0$ where $\mathrm{D}_{a} u$ denotes the absolutely continuous part of the measure $\mathrm{D} u$. Let $\Gamma$ be the support of the singular part of $\mathrm{D} \bar{u}$. Therefore, $\bar{u}$ is piecewise constant on $] a, b[\backslash \Gamma$.

We have also a similar result for the 2D-case. Assume that

$\forall \mathcal{U}$ open subset of $\Omega, u_{d \mid \mathcal{U}}$ is not equal to a $\mathrm{BV}(\Omega)$ function, then $u_{d}-\bar{v}$ satisfies $\left(\mathcal{H}_{2}\right)$ as well (since $\bar{v} \in \mathrm{W}^{1,1}(\Omega)$ ). Following Proposition 6 of [32], there is no open subset $\omega$ of $\Omega$ on which both components $\frac{\partial \bar{u}}{\partial x_{i}}, i=1,2$ have constant, non-zero sign. 


\subsection{Uniqueness}

The functional $\mathcal{F}_{\lambda, \mu}$ is convex but not strictly convex, because of the degenerating direction $u+v=0$. It is obvious that, if $\left(u^{*}, v^{*}\right)$ is a solution, then $\left(u^{*}+c, v^{*}-c\right)$ (where $c$ is constant), is a solution as well. Let us call

$$
\mathbf{C}(\Omega):=\left\{(u, v) \in \operatorname{BV}(\Omega) \times \mathrm{BH}_{0}(\Omega): \exists c \in \mathbb{R} u=c \text { and } v=-c \text { a.e on } \Omega\right\} .
$$

The question of uniqueness reduces to uniqueness up to $\mathbf{C}(\Omega)$ functions. In other words, if $\left(u_{1}, v_{1}\right)$ and $\left(u_{2}, v_{2}\right)$ are two optimal solutions of $\left(\mathcal{P}_{\lambda, \mu}\right)$ can we show that $u_{2}=u_{1}+c$ and $v_{2}=v_{1}-c$ where $c$ is a constant function? It is still an open problem for the $2 \mathrm{D}$ case. This point has been discussed more precisely in [31]. Nevertheless, we may give partial results:

Proposition 4.1 Assume $\left(u_{1}, v_{1}\right)$ and $\left(u_{2}, v_{2}\right)$ are two optimal solutions of $\left(\mathcal{P}_{\lambda, \mu}\right)$. Then, there exists $\varphi \in \mathrm{BV}(\Omega) \cap \mathrm{BH}_{0}(\Omega)$ such that $u_{2}=u_{1}-\varphi$ and $v_{2}=v_{1}+\varphi$.

Proof. Set $u=u_{2}-u_{1}(\in \mathrm{BV}(\Omega))$ and $v=v_{2}-v_{1}\left(\in \mathrm{BH}_{0}(\Omega)\right)$.

As $u_{d}-u_{1}-v_{1}=u_{d}-u_{2}-v_{2}$ (this is the unique solution of the dual problem), then $u+v=0$. This yields that $u=-v \in \mathrm{BV}(\Omega) \cap \mathrm{BH}_{0}(\Omega)$ and we get the result.

Lemma 4.2 The only solutions $(\bar{u}, \bar{v})$ to $\left(\mathcal{P}_{\lambda, \mu}\right)$ that satisfy $\bar{u}+\bar{v}=0$ are functions of $\mathbf{C}(\Omega)$.

Proof. If we assume that $\bar{u}+\bar{v}=0$, then $\bar{u} \in \mathrm{BH}_{0}(\Omega)$ on one hand and $\Phi^{2}(\bar{v})=\Phi^{2}(-\bar{u})=\Phi^{2}(\bar{u})$ on the other hand. As $\mathcal{F}_{\lambda, \mu}(\bar{u}, \bar{v}) \leq \mathcal{F}_{\lambda, \mu}(u, v)$, for every $(u, v) \in \mathrm{BV}(\Omega) \times \mathrm{BH}_{0}(\Omega)$ this yields

$\left\|u_{d}\right\|_{\mathrm{L}^{2}(\Omega)}^{2}+2 \lambda \mathrm{TV}(\bar{u})+2 \mu \mathrm{TV}^{2}(\bar{u}) \leq\left\|u_{d}-u-v\right\|_{\mathrm{L}^{2}(\Omega)}^{2}+2 \lambda \mathrm{TV}(u)+2 \mu \mathrm{TV}^{2}(v)$.

Taking $u=v=0$ gives

$$
\left\|u_{d}\right\|_{\mathrm{L}^{2}(\Omega)}^{2}+2 \lambda \mathrm{TV}(\bar{u})+2 \mu \mathrm{TV}^{2}(\bar{u}) \leq\left\|u_{d}\right\|_{\mathrm{L}^{2}(\Omega)}^{2} .
$$

So we get $\lambda \operatorname{TV}(\bar{u})+\mu \operatorname{TV}^{2}(\bar{u})=0$. This implies that $\operatorname{TV}(\bar{u})=0$, so that $\bar{u}$ is a constant function.

Theorem 4.2 Let $(\lambda, \mu)$ be nonnegative real numbers such that $\lambda \geq\left\|u_{d}\right\|_{G}$ and $\mu \geq C_{2} \lambda$, where $C_{2}$ is the constant of Lemma 2.1. Then the $\mathbf{C}(\Omega)$ functions are the only solutions to $\left(\mathcal{P}_{\lambda, \mu}\right)$.

Proof. Let us assume that $\lambda \geq\left\|u_{d}\right\|_{G}$ and $\mu \geq C_{2} \lambda$, where $C_{2}$ is the constant of Lemma 2.1. Lemma 4.1 gives

$$
\forall(u, v) \in \mathrm{BV}(\Omega) \times \mathrm{BH}_{0}(\Omega) \quad\left|\left(\mathrm{u}_{\mathrm{d}}, \mathrm{u}+\mathrm{v}\right)_{2}\right| \leq \lambda \mathrm{TV}(\mathrm{u}+\mathrm{v}),
$$

since $u_{d} \in \mathrm{L}^{2}(\Omega)$ and $\mathrm{BH}_{0}(\Omega) \subset \mathrm{BV}(\Omega)$. Then

$$
\left|\left(u_{d}, u+v\right)_{2}\right| \leq \lambda \operatorname{TV}(u)+\lambda \operatorname{TV}(v) .
$$


Lemma 2.1 gives a constant $C_{2}$ (only depending on $\Omega$ ) such that

$$
\forall v \in \mathrm{BH}_{0}(\Omega) \quad \mathrm{TV}(v) \leq C_{2} \mathrm{TV}^{2}(v)
$$

so that for every pair $(u, v) \in \mathrm{BV}(\Omega) \times \mathrm{BH}_{0}(\Omega)$

$$
\left|\left(u_{d}, u+v\right)_{2}\right| \leq \lambda \mathrm{TV}(u)+C_{2} \lambda \mathrm{TV}^{2}(v) \leq \lambda \mathrm{TV}(u)+\mu \mathrm{TV}^{2}(v)
$$

Finally, we get for every $(u, v) \in \mathrm{BV}(\Omega) \times \mathrm{BH}_{0}(\Omega)$

$$
\begin{aligned}
& \frac{1}{2}\left\|u_{d}\right\|^{2}=\frac{1}{2}\left\|u_{d}-u-v\right\|^{2}-\frac{1}{2}\|u+v\|^{2}+\left(u_{d}, u+v\right)_{2} \\
\leq & \frac{1}{2}\left\|u_{d}-u-v\right\|^{2}+\lambda \operatorname{TV}(u)+\mu \operatorname{TV}^{2}(v) .
\end{aligned}
$$

This means that $\mathcal{F}_{\lambda, \mu}(0,0) \leq \mathcal{F}_{\lambda, \mu}(u, v)$ : so $(0,0)$ is a solution to $\left(\mathcal{P}_{\lambda, \mu}\right)$. Let $(\bar{u}, \bar{v}) \in \mathrm{BV}(\Omega) \times \mathrm{BH}_{0}(\Omega)$ be another solution to $\mathcal{P}_{\lambda, \mu}$. With Proposition 4.1, we get $\bar{u}+\bar{v}=0$ and Lemma 4.2 gives $(\bar{u}, \bar{v}) \in \mathbf{C}(\Omega)$.

Remark 4.2 The previous theorem tells that if $\frac{\mu}{\lambda}$ and $\lambda$ are large enough then the set of solutions is $\mathbf{C}(\Omega)$. In addition, if we impose (for example) that $u \in \mathrm{G}(\Omega)$ (that is $u$ has a null mean value), then the unique solution is $(0,0)$ since $\mathbf{C}(\Omega) \cap\left(\mathrm{G}(\Omega) \times \mathrm{BH}_{0}(\Omega)\right)=\{(0,0)\}$.

Eventually, we have a uniqueness result for the $1 \mathrm{D}$ case:

Theorem 4.3 Assume $n=1, \Omega=] a, b\left[\right.$ and that $u_{d}$ satisfies assumption $\left(\mathcal{H}_{1}\right)$. Then, for every $\lambda>0, \mu>0$ problem $\left(\mathcal{P}_{\lambda, \mu}\right)$ has a unique solution up to a $\mathbf{C}(\Omega)$ function.

More precisely, if $\left(u_{1}, v_{1}\right)$ and $\left(u_{2}, v_{2}\right)$ are two optimal solutions of $\left(\mathcal{P}_{\lambda, \mu}\right)$ then $\varphi:=u_{2}-u_{1}=v_{2}-v_{1}$ is a constant function. In particular, problem $\left(\mathcal{P}_{\lambda, \mu}\right)$ has a unique solution $\left(u^{*}, v^{*}\right)$ such that $u^{*}$ has a null mean value.

Proof. Let $\left(u_{1}, v_{1}\right)$ and $\left(u_{2}, v_{2}\right)$ be two optimal solutions of $\left(\mathcal{P}_{\lambda, \mu}\right)$. With Proposition 4.1, there exists $\varphi \in \mathrm{BV}(\Omega) \cap \mathrm{BH}_{0}(\Omega)$ such that $\varphi=u_{2}-u_{1}=v_{2}-v_{1}$. If $u_{d}$ satisfies $\left(\mathcal{H}_{1}\right)$, then $u_{d}-v_{i}, i=1,2$ obviousy satisfies this assumption as well. As $u_{i}, i=1,2$ is solution to the first order Rudin-Osher-Fatemi problem

$$
u_{i}=\operatorname{argmin}\left\{\frac{1}{2}\left\|u_{d}-v_{i}-u\right\|^{2}+\Phi_{\lambda}^{1}(u): u \in \mathrm{L}^{2}(\Omega)\right\}, i=1,2
$$

(see [1]), then $u_{1}, u_{2}$ and $\varphi$ are piecewise constant on $\Omega$.

In addition $\varphi=v_{2}-v_{1} \in \mathrm{BH}(\Omega) \subset \mathrm{W}^{1,1}(\Omega)$. This implies that $\varphi$ is continuous and proves it is constant. 


\section{Perspectives}

The presented model seems promising for texture extraction and/or texture analysis. However, there are still many open problems. The uniqueness of solutions (up to a constant function) is a crucial issue (with respect to the numerical computation. We have observed in [31] that solutions are unique up to a constant : this constant depends on the initialization process. However, we only have partial theoretical results as shown in the previous section.

The second important (open) question concerns the regularity/ behavior of the solution. More precisely, we infer that the BV-part $u$ is piecewise constant if the parameters $\lambda$ and $\mu$ are large enough. So, we would like to prove that $u \in \operatorname{SBV}(\Omega) \backslash \mathrm{W}^{1,1}(\Omega)$. To achieve this goal, we have to describe carefully the derivative $\mathrm{D} u$ of $u$, especially the singular part. We conjecture that the contours are completely described by this singular part. More precisely, we would like to prove that $u=\sum_{i=1}^{N} u_{i} \chi_{\Omega_{i}}$, where

- $\Omega_{i}, i=1, \cdots, N$ are open subsets of $\Omega$ such that $\bigcup_{i=1}^{N} \operatorname{cl}\left(\Omega_{i}\right)=\operatorname{cl}(\Omega)$,

- $\chi_{A}$ is the characteristic function of the set $A: \chi_{A}(x)=1$ if $x \in A, 0$ else.

- $u_{i} \in \mathrm{W}^{1,1}\left(\Omega_{\mathrm{i}}\right), i=1, \cdots, N$, and if possible $u_{i}$ is a constant function for every $i$.

In this case, we would have $\bigcup_{i=1}^{N} \partial \Omega_{i}$ as the contour set. Once again, we have given partial results that deserve to be completed, especially in the $2 \mathrm{D}$ case.

The last important issue is to perform a sensitivity analysis of the solution structure with respect to parameters $\lambda$ and $\mu$. The question has been studied in [31] from a numerical point of view, with many examples. The questions that arise are

- the quantification of the ratio $\frac{\mu}{\lambda}$ (see Theorem 4.2),

- the proof of the properties that has been observed numerically. More precisely:

- in the case where the data is noiseless or not too much textured, the decomposition given by $\lambda \precsim \mu$ and the initialization $u_{0}=v_{0}=0$, gives a cartoon part which is piecewise constant. In this case, the remainder $\mathrm{L}^{2}$ term is the texture and/or noise.

- in the case where the image is highly textured, the model provides a two-scale decomposition. The TV-part represents the macro-texture and the $\mathrm{L}^{2}$ - part the micro-texture and/or noise. The scaling is tuned via the ratio $\rho=\frac{\lambda}{\mu}$. We have to make this point precise.

- the proof that the decomposition is always the same for any $\mu>>\lambda$, once $\lambda$ has been chosen. 
- the estimates of suitable parameters with respect to the original image properties (as the G-norm, $\mathrm{L}^{2}$-norm etc.)

- the behavior of $\|w\|_{L^{2}}, \operatorname{TV}(u), \operatorname{TV}^{2}(v)$, with respect to $\lambda$ and $\mu$.

\section{Conclusions}

This model is well adapted to texture extraction and analysis. We have proved existence and partial uniqueness results using inf-convolution tools and convex duality. The decomposition we obtain can be used as a preprocessing tool. Indeed, each part can be separately studied to get different informations for the original image.

- The $\mathrm{L}^{2}$-part is the oscillating part (it belongs to the Meyer space). It models gaussian noise if the image is noisy and/or involves micro-textures. Removing this part from the original image gives a denoising method that preserves contours, without any staircasing effect (no additional false contours). Moreover, one can focus on this part to precisely analyze its structure (random or not) to separate noise from texture.

- The BV-part represents the cartoon part : even if it is not piecewise constant, the jump set gives the contours of the image. We may use classical segmentation methods to deal with this component.

- The BH- part is continuous (at least for $d \leq 2$ ). It gives the image dynamic. It is useful to get rid of lightning perturbations, for example.

\section{References}

1. L.I. Rudin, S. Osher, and E. Fatemi. Nonlinear total variation based noise removal algorithms. Physica D, 60:259-268, 1992.

2. L. Ambrosio, N. Fusco, and D. Pallara. Functions of bounded variation and free discontinuity problems. Oxford Mathematical Monographs. The Clarendon Press Oxford University Press, New York, 2000.

3. H. Attouch, G. Buttazzo, and G. Michaille. Variational analysis in Sobolev and BV spaces, volume 6 of MPS/SIAM Series on Optimization. Society for Industrial and Applied Mathematics (SIAM), Philadelphia, PA, 2006. Applications to PDEs and optimization.

4. L.C. Evans and R. Gariepy. Measure theory and fine properties of functions. CRC Press, 1992.

5. G. Aubert and J.-F. Aujol. Modeling very oscillating signals. Application to image processing. Appl. Math. Optim., 51(2):163-182, 2005.

6. J.-F. Aujol, G. Aubert, L. Blanc-Féraud, and A. Chambolle. Image decomposition into a bounded variation component and an oscillating component. J. Math. Imaging Vision, 22(1):71-88, 2005

7. S. Osher, A. Sole, and Vese L. Image decomposition and restoration using total variation minimization and the $\mathrm{H}^{1}$ norm. SIAM Journal on Multiscale Modeling and Simulation, 1-3(349-370), 2003.

8. S. Osher and L. Vese. Modeling textures with total variation minimization and oscillating patterns in image processing. Journal of Scientific Computing, 19(1-3):553-572, 2003.

9. S. Osher and L. Vese. Image denoising and decomposition with total variation minimization and oscillatory functions. special issue on mathematics and image analysis. J. Math. Imaging Vision,, 20(1-2):7-18, 2004. 
10. W. Yin, D. Goldfarb, and S. Osher. A comparison of three total variation based texture extraction models. J. Vis. Commun. Image, 18:240-252, 2007.

11. M. Bergounioux and L. Piffet. A second-order model for image denoising. Set-Valued and Variational Analysis, 18(3-4):277-306, 2010.

12. M. Bergounioux and L. Piffet. A full second order variational model for multiscale texture analysis. Computational Optimization and Applications, 54:215-237, 2013.

13. F. Demengel. Fonctions à hessien borné. Annales de l'institut Fourier, 34(2):155-190, 1984.

14. M. Bergounioux. On Poincaré-Wirtinger inequalities in BV-spaces. Control \& Cybernetics, 4(40):921-929, 2011.

15. K. Bredies, K. Kunisch, and T. Pock. Total generalized variation. SIAM J. Imaging Sci., 3(3):492-526, 2010.

16. K. Bredies, K. Kunisch, and T. Valkonen. Properties of $l^{1}-\operatorname{tgv}^{2}$ : the one-dimensional case. J. Math. Anal. Appl., 398(1):438-454, 2013.

17. K. Bredies and M Holler. Regularization of linear inverse problems with total generalized variation. Journal of Inverse and Ill-posed Problems, 68, 2014.

18. L. Ambrosio, L. Faina, and R. March. Variational approximation of a second order free discontinuity problem in computer vision. SIAM J. Math. Anal., 32(6):1171-1197, 2001.

19. M. Carriero, A. Leaci, and F. Tomarelli. Uniform density estimates for the blake \& zisserman functional. Discrete and Continuous Dynamical Systems, 31(4):1129-1150, 2011.

20. M. Carriero, A. Leaci, and F. Tomarelli. Free gradient disconitnuity and image inpainting. J. Math. Sciences, 181(6):805-819, 2012.

21. Y. Meyer. Oscillating Patterns in Image Processing and Nonlinear Evolution Equations, volume 22 of University Lecture Series. AMS, 2001.

22. A. Chambolle and P.-L. Lions. Image recovery via total variation minimization and related problems. Numer. Math., 76:167-188, 1997.

23. G. Aubert and P. Kornprobst. Mathematical Problems in Image Processing, Partial Differential Equations and the Calculus of Variations, volume 147 of Applied Mathematical Sciences. Springer Verlag, 2006.

24. W.P. Ziemer. Weakly Differentiable Functions - Sobolev Space and Functions of Bounded Variation. Springer, 1980.

25. M. Carriero, A. Leaci, and F. Tomarelli. Special bounded hessian and elatsic-plastic plate. Rend. Ac. Naz delle Scienze, XVI(13):223-258, 1992.

26. J.-J. Moreau. Inf-convolution des fonctions numériques sur un espace vectoriel. Comptes Rendus de lAcadémie des Sciences de Paris,, 256:50475049, 1963.

27. J.-B. Hiriart-Urruty and R.R. Phelps. Subdifferential calculus using $\varepsilon$-subdifferentials. Journal of Functional Analysis, 118:150-166, 1993.

28. I Ekeland and R. Temam. Convex Analysis and Variational problems. SIAM Classic in Applied Mathematics, 28, 1999.

29. A. Chambolle. An algorithm for total variation minimization and applications. Journal of Mathematical Imaging and Vision, 20:89-97, 2004.

30. V. Caselles, A. Chambolle, and M. Novaga. The discontinuity set of solutions of the tv denoising problem and some extensions. SIAM Multiscale Model. Simul., 6(3):879-894, 2007.

31. M. Bergounioux. Inf-convolution model : numerical experiment. Technical report, hal.archives-ouvertes.fr/hal-01002958v2, 2014.

32. W. Ring. Structural properties of solutions of total variation regularization problems. ESAIM, Math Modelling and Numerical Analysis, 34:799 -840, 2000. 